\title{
Effects of tai chi qigong on psychosocial well-being among hidden elderly, using elderly neighborhood volunteer approach: a pilot randomized controlled trial
}

\section{Aileen WK Chan \\ Doris SFYu \\ KC Choi}

The Nethersole School of Nursing, Faculty of Medicine, The Chinese University of Hong Kong, Shatin, NT, Hong Kong SAR
Correspondence: Aileen WK Chan The Nethersole School of Nursing, Faculty of Medicine, 7/F, Esther Lee Building, The Chinese University of Hong Kong, Shatin, NT, Hong Kong SAR

$\mathrm{Tel}+85239434290$

Fax +85226035269

Email aileenchan@cuhk.edu.hk
This article was published in the following Dove Press journal:

Clinical Interventions in Aging

5 January 2017

Number of times this article has been viewed

Purpose: To test the feasibility and preliminary effectiveness of a tai chi qigong program with the assistance of elderly neighborhood volunteers in strengthening social networks and enhancing the psychosocial well-being of hidden elderly.

Patients and methods: "Hidden elderly" is a term used to describe older adults who are socially isolated and refuse social participation. This pilot randomized controlled trial recruited 48 older adults aged 60 or above who did not engage in any social activity. They were randomized into tai chi qigong $(n=24)$ and standard care control $(n=24)$ groups. The former group underwent a three-month program of two 60-minute sessions each week, with the socially active volunteers paired up with them during practice. Standard care included regular home visits by social workers. Primary outcomes were assessed by means of the Lubben social network and De Jong Gieveld loneliness scales, and by a revised social support questionnaire. Secondary outcomes were covered by a mental health inventory and the Rosenberg self-esteem scale, and quality of life by using the 12-Item Short Form Health Survey. Data was collected at baseline, and at three and six months thereafter.

Results: The generalized estimating equations model revealed general improvement in outcomes among participants on the tai chi qigong program. In particular, participants reported a significantly greater improvement on the loneliness scale $(B=-1.32,95 \%$ confidence interval $[\mathrm{CI}]-2.54$ to $-0.11, P=0.033)$ and the satisfaction component of the social support questionnaire $(B=3.43,95 \%$ CI $0.10-6.76, P=0.044)$ than the control group.

Conclusion: The pilot study confirmed that tai chi qigong with elderly neighborhood volunteers is a safe and feasible social intervention for hidden elderly. Its potential benefits in improving social and psychological health suggest the need for a full-scale randomized controlled trial to reveal its empirical effects.

Keywords: group-based activity, social isolation, social network, social support

\section{Clinical trial registry}

Clinical trial registry: CUHK_CCRB00489 (https://www2.ccrb.cuhk.edu.hk/registry/ public/343).

\section{Introduction}

With the life expectancy of older people rising, the world population aged 60 and older is expected to increase from 900 million in 2015 to 2 billion by 2050 , with 400 million aged 80 and over. ${ }^{1}$ Projections indicate that nearly one-third of the local population will 
be 65 or above by $2040 .^{2}$ More than 400,000 elderly people live alone or only with a spouse, and this number accounts for $37.4 \%$ of the elderly population in Hong Kong. Older people often have fewer social contacts and feel lonely because they have a poor network of family or friends. ${ }^{3}$ Some older adults are disengaged or "hidden" from the community. "Hidden" has no strict definition, but the term generally refers to older people who are disengaged from society, do not know how to seek help or support, and are not known to the existing community support network. ${ }^{4}$ The exact number of such hidden elderly people in the community is not known, but research conducted in 2009 estimated 70,000-90,000 "hidden elderly" in Hong Kong. ${ }^{5}$ Most of these older people have a feeling of being discriminated against by neighbors, and are reluctant to establish contact with outsiders. Consequently, they become the hidden elderly. ${ }^{6}$

The local literature reports that the hidden elderly have weak family and friends network support. ${ }^{6}$ They feel isolated, lonely, and neglected. The factors contributing to their hidden life are associated with the aging process, such as retirement, declining health condition, widowhood, and conflict with their family. ${ }^{6}$ The hidden elderly are lacking in financial resources and knowledge of how to seek outside help. In Hong Kong, outreach social services have been set up to identify such older people in the community, provide home-based counseling, and connect them to center-based elderly services. However, there is little evidence of the impact of such services on the social health and service utilization of the hidden elderly.

Social isolation is an important health concern for such people, as they usually live alone and enjoy little social participation. ${ }^{7}$ There is evidence that socially isolated individuals have two to four times greater risk of all-cause mortality than elderly people who have more friends, relatives, and neighbors. ${ }^{8}$ Engaging the hidden elderly in meaningful social life and health promotion activities is therefore seen as an agenda to be highly prioritized in elderly services. ${ }^{9}{ }^{10}$ Group-based physical activity may be an effective intervention, and the literature indeed reveals that such activity is more effective than one-to-one interventions in preventing social isolation and depression by improving social networking with other participants. ${ }^{11,12}$ Studies have also indicated that when physical activity is performed in a group, social reinforcement from participation can have social benefits to counter social isolation and create friendships. ${ }^{13}$ Tai chi qigong exercise is a popular physical activity intervention among older adults, with a high level of perceived benefit. ${ }^{14-16}$ It is usually performed in groups, and has a high acceptability rating among frailer elderly. ${ }^{17}$
All these characteristics suggest the potential value of tai chi qigong in improving the social health and quality of life of hidden elderly in the community.

\section{Tai chi qigong}

The foundation of tai chi qigong is a combination of exercise and meditation. Qigong is an ancient Chinese system of gentle self-healing exercises, while tai chi is a martial art that can be viewed as a therapeutic exercise based on traditional Chinese medicine theory. Tai chi and qigong can be combined to enhance the training effect, and form an activity that engages the mind and the body, the engagement of the mind particularly leading to meditative quality and preventing boredom. ${ }^{18}$ Research has demonstrated that tai chi and qigong have beneficial effects on patients with respiratory disease, ${ }^{13,19}$ heart disease, ${ }^{20,21}$ metabolic control in type-2 diabetes, ${ }^{22}$ high blood pressure, ${ }^{23}$ emotional stress, ${ }^{24}$ and sleep disturbances in older adults. ${ }^{25}$ With the favorable results of previous studies, the applicably of tai chi qigong to the hidden elderly deserves special attention.

\section{Elderly neighborhood volunteers}

The major challenge in planning an intervention program for the hidden elderly is how to motivate them to participate. Although they are a socially isolated group, a previous study found that $75 \%$ of the hidden elderly welcomed being contacted, helped out, and cared for. ${ }^{5}$ Aside from household members, neighbors are their most proximate social contacts. Mobilizing elderly neighborhood volunteers to motivate and strengthen the social networks of the hidden elderly can be a useful strategy. Neighborhood volunteering in the US has shown itself able to strengthen the community through enhancement of neighborliness and volunteerism..$^{26,27}$ Having strong ties to neighbors facilitates access to immediate and informal aid as well as reducing the sense of isolation. ${ }^{27}$

The present study aims to test the feasibility and preliminary effectiveness of a peer neighborhood-assisted tai chi qigong program in strengthening social networks and enhancing the psychosocial well-being of hidden elderly.

\section{Materials and methods Study design}

The study is a single-blind randomized controlled trial (RCT). Participants were allocated to the tai chi qigong group or a standard care control group.

\section{Subjects and venues}

The study was conducted in a district elderly community center (DECC) operated by a nongovernment organization 
from January 2016 to August 2016. Subjects were included if they (1) were aged 60 or above, and (2) did not engage in any social activities. They were excluded if they had (1) some cognitive impairment (abbreviated mental test score $<7$ ) or (2) musculoskeletal disorders or other disabling diseases that might limit the practice of tai chi qigong.

\section{Sample size and randomization}

Considering the general rule of thumb covering sample size requirements for a pilot study, ${ }^{28}$ a total of 48 subjects were recruited. They were randomly allocated to either the intervention $(n=24)$ or the control group $(n=24)$ in a 1:1 ratio according to a computer-generated random sequence list prepared by an independent statistician. The grouping sequence list was password-protected and stored on a computer. Only authorized staff responsible for group allocation were allowed access to the list. Group allocation of each recruited participant was assigned in accordance with his/her sequence of enrollment and the corresponding group identifier in the previously prepared random grouping list.

\section{Subject recruitment procedure and randomization methods}

The nurse and social worker in the study setting helped to identify potential subjects, and their eligibility was screened by the research assistant (RA). The subjects were clearly informed about the purpose and procedures of the study, and each one was given an opportunity to ask questions. A written informed consent was obtained from each eligible person agreeing to join the study.

\section{Elderly volunteers}

A total of eight active senior volunteers were invited by the social worker in the DECC to join the program. Volunteers were included if they were aged 55 or above, cognitively intact, active in social activities, and willing to help others. They were excluded if they were physically unstable or suffered from impaired mobility.

\section{Intervention protocol}

\section{Training of the elderly volunteers}

The volunteers received training from an experienced nurse and a social worker from the DECC. The training focused on (1) issues of social isolation, (2) building up trust and bonding with others, (3) interpersonal skills in interacting with the hidden elderly, and (4) safety issues and general care of such people. Four weekly training sessions were conducted, each consisting of two-hour training in the form of seminar, workshop, and Q \& A period. After completing the training sessions, the volunteers were recognized as "health ambassadors" for this project. The ambassadors were neighbors or those who lived in the same building as the hidden elderly. The proximity of the matched ambassadors and hidden elderly helped the latter to develop a sense of neighborhood, build up peer relationships, and be aware that immediate help was available whenever necessary. ${ }^{27}$

\section{Tai chi qigong group}

Participants in the tai chi qigong group attended a 60-minute exercise class twice a week for three months. The intervention program included 18 forms of tai chi qigong (Table S1). The 18 forms were chosen to emphasize elements of breathing, range of motion on limbs, and mind-body coordination. Participants were required to coordinate their breathing with the slow and graceful movement motions. The classes were held in a DECC activity room. The participants were led by an experienced tai chi qigong instructor, whose motions, postures, and speed of movement they had to copy. Any incorrect performance was rectified by the instructor in the class. The number of participants in each session was limited to fewer than 15, which was considered manageable for teaching and learning. An RA was present at each session to monitor the consistency of the exercise practice, using a skill competence checklist.

Participants were encouraged to self-practice tai chi qigong for 30 minutes every day. Pictures of the 18 forms of the exercise, printed on A3 paper, were provided to each participant to help his/her self-practice, and served as an important reminder.

The main role of the health ambassadors was to act as facilitators for the tai chi qigong group. As they were neighbors of the hidden elderly, it was convenient for them to remind the participants to attend classes and accompany them there. They would then form a self-practice group with three participants, each ambassador acting as the group leader. The ambassadors were expected to influence the participants to join the self-practice group and accompany them to exercise in the park every day. They also recorded the frequency of the participants' daily self-practice - a more objective and reliable system than the self-recorded logbook for monitoring subject compliance.

For ethical reasons, the ambassadors were instructed not to coerce the subjects into participating, but to use a friendly and caring approach to encouraging them to attend the tai chi qigong classes and self-practice groups. They were reminded that participation was voluntary and that subjects should not be forced into it in any way. 


\section{Control group}

The subjects in the control group received usual care, which included irregular home visits conducted by social workers ranging from monthly to quarterly. They also received regular information of community services mailed to them monthly. No additional activity was recommended to the control group.

\section{Outcome measurement}

\section{Primary outcomes: social support outcomes}

Social network measurement - the Lubben social network scale (LSNS-6)

The six-item LSNS-6 assesses the size of the active social network among community-dwelling older adults on a six-point Likert scale. Total scores range from 0 to 30 , with higher scores indicating larger social networks. The validated LSNS-6 has established a cut-off point of 12 for best overall sensitivity. ${ }^{29,30}$ The scale has been widely used in elderly populations. ${ }^{31}$

\section{Feelings of loneliness - the De Jong Gieveld} loneliness scale

The six-item scale is widely used to measure loneliness. Cronbach's $\alpha$ on the scale is 0.76 . Intraclass correlation coefficients range from 0.98 to $1.00 .{ }^{32}$ The scale has proved to be a reliable and valid instrument to measure overall, emotional, and social loneliness.

\section{Revised social support questionnaire (RSSQ)}

The RSSQ is a six-item questionnaire designed to measure social support. Each item includes a question with two parts, assessing the number of people in contact and the level of satisfaction with these people perceived by the subject. The intraclass correlations for parts 1 and 2 are 0.90 and 0.93 respectively. ${ }^{33}$

\section{Secondary outcomes: psychosocial outcomes}

Mental health status - the mental health

inventory (MHI- 18)

The MHI-18 assesses several domains of the subject's mental health status, such as anxiety, depression, behavioral control, positive affect, and general distress. The reliability estimates range from 0.83 to 0.91 for scales based on five lower-order factors, and from 0.92 to 0.96 for those based on two higherorder factors. ${ }^{34}$

\section{Rosenberg self-esteem scale}

This scale, measuring self-esteem, is a ten-item Likert type, with items answered on a four-point scale where higher levels of self-esteem are indicated by higher scores. Cronbach's $\alpha$ is $0.79 .{ }^{35}$

Quality of life - 12-Item Short Form Health Survey (SF-I2) The SF-12 is a popular generic measure of health-related quality of life. The 12-item questionnaire measures functional health and well-being from the client's perspective. The survey has favorable internal consistency and test-retest reliability (from 0.67 to 0.82 ). ${ }^{36}$

\section{Data processing and analysis}

To minimize data collection bias, the RAs responsible for data collection were blinded to the subject group allocation. They attended a briefing session where data collection procedures were explained. Demographic data and baseline measurements for both groups were collected at the beginning of the study (T0). Post-intervention and follow-up data was collected at three months (T1) and six months (T2).

\section{Process evaluation}

A self-developed survey was used with the tai chi qigong group at the three-month mark to evaluate subjects' perceptions of and satisfaction with the program.

\section{Data analysis}

IBM SPSS 22.0 (IBM Corp., Armonk, NY, USA) was used for statistical analysis. Appropriate descriptive statistics were used to present the characteristics of the subjects. Baseline background characteristics of the two groups were compared by independent $t$, chi-square, or Fisher's exact tests, as appropriate. Generalized estimating equations (GEE) models with appropriate link functions were used to compare the repeated measures of primary and secondary outcomes in the intervention and control groups across the time points. Statistical analyses were conducted based on the intention-to-treat principle. The GEE model could account for intra-correlated repeated measures data and accommodate missing data caused by incomplete visits or dropout in RCT. ${ }^{37}$ A dummy variable (group) was assigned to represent the intervention group, with the control group as reference. Other dummy variables, namely $\mathrm{T} 1$ and $\mathrm{T} 2$, were used to represent time differences corresponding to $\mathrm{T} 1$ and $\mathrm{T} 2$, with the baseline $\mathrm{T} 0$ as reference. The interaction terms of the time-point and group dummy variables (group*T1 and group*T2) were included in the GEE model to assess the differential changes in the outcomes of intervention and control groups at $\mathrm{T} 1$ and T2 relative to T0. All statistical tests were two-sided, with the significance level set at 0.05 . 


\section{Ethical considerations}

Ethical approval was obtained from the Joint Clinical Research Ethics Committee of the Chinese University of Hong Kong and New Territory East Cluster. Permission to conduct the study in the selected community center was also obtained. The subjects participated voluntarily in the study, and were informed of its purposes. A written informed consent was obtained from every eligible subject.

\section{Results}

To complete the enrollment process for the study, a total of 57 people were screened. Seven did not fulfill the inclusion criteria, and two declined to participate. A total of 48 were recruited and randomly assigned to the tai chi qigong $(n=24)$ or control group $(n=24)$. Two subjects in the control group did not complete the baseline assessment, and were therefore excluded from the analysis. The age of subjects ranged from 66 to 103 years, with a mean of 77.3 years (standard deviation [SD] =7.4). Of the 46, 35 (76\%) were female, and 11 (24\%) male. The first and second follow-up assessments were completed by 41 and 35 subjects, respectively (Figure 1).

The baseline characteristics of the subjects in both groups are listed in Table 1. The groups were essentially comparable, and no statistically significant differences were observed. The average age of the tai chi qigong group was 75.4 years $(\mathrm{SD}=5.9)$, and of the control 79.4 years $(\mathrm{SD}=8.5)$.

The primary and secondary outcomes across the study time points (T0 or baseline, T1, and T2) of the two groups are given in Table 2. The GEE model was used to compare the differential changes from $\mathrm{T} 0$ of each of the outcomes at T1 and T2 (Table 3). The GEE analysis showed that the tai chi qigong group had more improvement at both $\mathrm{T} 1$ and

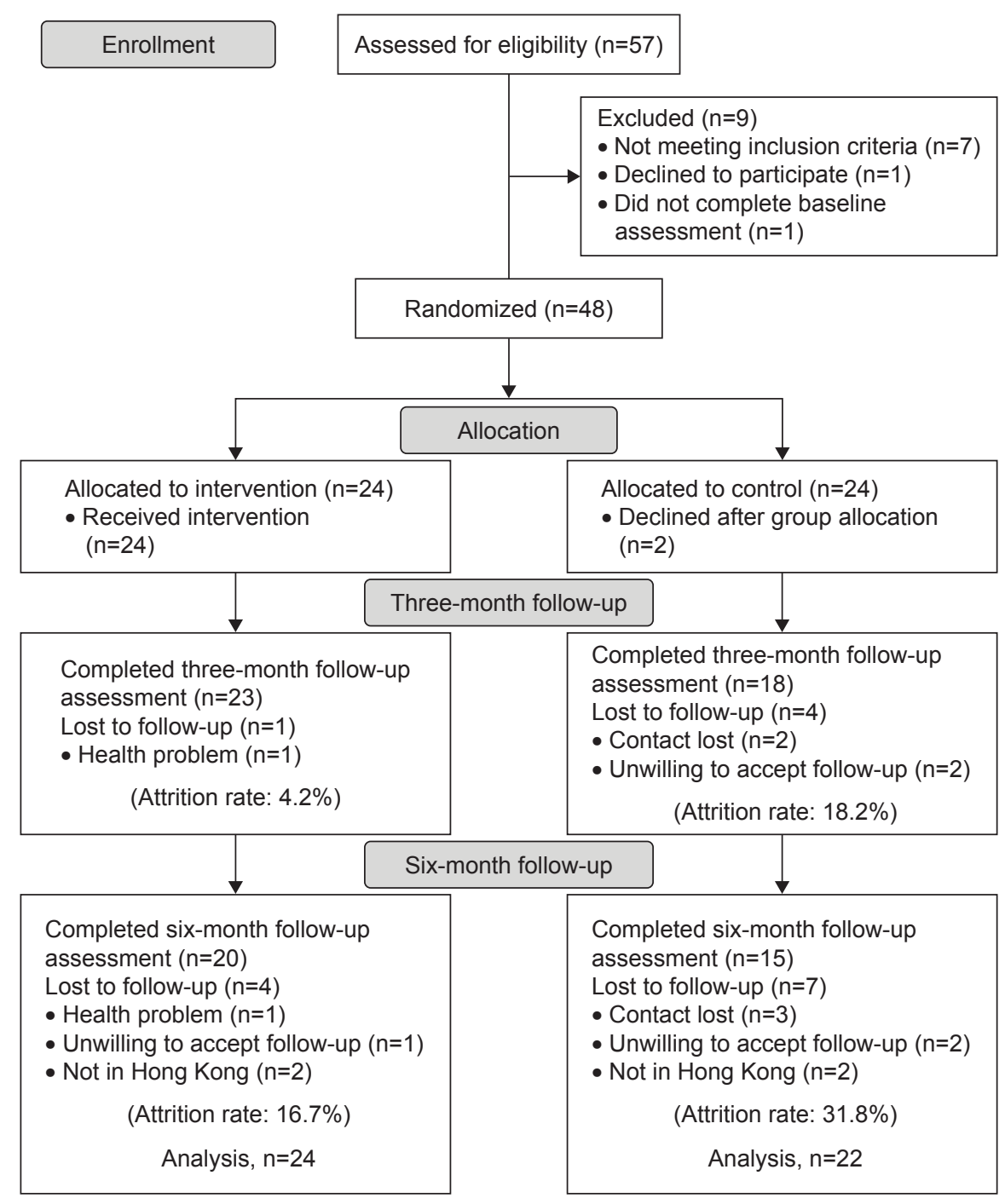

Figure I Flowchart tracking participants through randomized controlled trial. 
Table I Baseline characteristics of the participants $(n=46)$

\begin{tabular}{|c|c|c|c|}
\hline Characteristics & $\begin{array}{l}\text { Control } \\
\text { group }(n=22)\end{array}$ & $\begin{array}{l}\text { Tai chi } \\
\text { group }(n=24)\end{array}$ & $P$-value $\#$ \\
\hline Age (years) & $79.4(8.5)$ & $75.4(5.9)$ & 0.071 \\
\hline Male & $5(22.7 \%)$ & $6(25.0 \%)$ & 0.857 \\
\hline Female & $17(77.3 \%)$ & $18(75.0 \%)$ & \\
\hline Single/separated/widowed & II (50.0\%) & $10(41.7 \%)$ & 0.571 \\
\hline Married & II (50.0\%) & $14(58.3 \%)$ & \\
\hline No & $14(63.6 \%)$ & 17 (70.8\%) & 0.603 \\
\hline Yes & $8(36.4 \%)$ & $7(29.2 \%)$ & \\
\hline \multicolumn{4}{|l|}{ Religious belief } \\
\hline No & $13(59.1 \%)$ & $10(41.7 \%)$ & 0.238 \\
\hline Yes & 9 (40.9\%) & $14(58.3 \%)$ & \\
\hline \multicolumn{4}{|l|}{ Smoking habits } \\
\hline Never smoked & I7 (77.3\%) & I 7 (70.8\%) & $0.999 \%$ \\
\hline Ex-smoker & $2(9.1 \%)$ & $3(12.5 \%)$ & \\
\hline Current smoker & $3(13.6 \%)$ & $4(16.7 \%)$ & \\
\hline \multicolumn{4}{|l|}{ History of hypertension } \\
\hline No & $5(22.7 \%)$ & $8(33.3 \%)$ & 0.425 \\
\hline Yes & $17(77.3 \%)$ & $16(66.7 \%)$ & \\
\hline \multicolumn{4}{|l|}{ History of diabetes } \\
\hline No & $13(59.1 \%)$ & $20(83.3 \%)$ & 0.068 \\
\hline Yes & 9 (40.9\%) & $4(16.7 \%)$ & \\
\hline \multicolumn{4}{|l|}{ History of cardiac disease } \\
\hline No & 20 (90.9\%) & 21 (87.5\%) & $0.999 \%$ \\
\hline Yes & $2(9.1 \%)$ & $3(12.5 \%)$ & \\
\hline $\begin{array}{l}\text { Weekly exercise, } \\
\text { duration in hours }{ }^{\ddagger}\end{array}$ & $3.5(1.2-6.0)$ & $2.8(1.2-4.0)$ & 0.349 \\
\hline
\end{tabular}

Notes: "Categorical and continuous variables were compared between the two groups using the Pearson chi-square test and Independent $t$-test, respectively. Data are presented as mean (standard deviation), n (\%), or median (interquartile range). $\psi \vee$ Variables were compared by Fisher’s exact test. $¥$ Variables were square roottransformed before being subjected to Independent $t$-test.

T2 with respect to T0 in all four social support outcomes. Statistically significant improvement was found on the De Jong Gieveld loneliness scale at T2 ( $B=-1.32$, 95\% confidence interval [CI] -2.54 to $-0.11, P=0.033$; Cohen's $d$ effect size 0.60 ) (Figure 2), and in the total satisfaction score at T2 on the RSSQ $(B=3.43,95 \%$ CI $0.10-6.76, P=0.044$; Cohen's $d$ effect size 0.76) (Figure 3). In the mental health inventory (MHI-18) outcomes, no significant improvement of the tai chi qigong group over the control group was noted, although there was more improvement in the total MHI-18 score. Regarding self-esteem and health-related quality-oflife outcomes, significant group-by-time interaction was only observed at $\mathrm{T} 1$ in the physical component score on SF-12 ( $B=7.69,95 \%$ CI 2.54-12.83, $P=0.003$; Cohen's $d$ effect size 0.87 ) (Figure 4).

The average attendance rate at the tai chi qigong classes was $71 \%$ (range $66 \%-100 \%$ ). Reasons for absence included medical follow-ups, forgetfulness, or illness. Most of the tai chi qigong participants (83\%) had managed to master the skills by the end of the program.
Table 2 Primary and secondary outcomes across time between the two groups

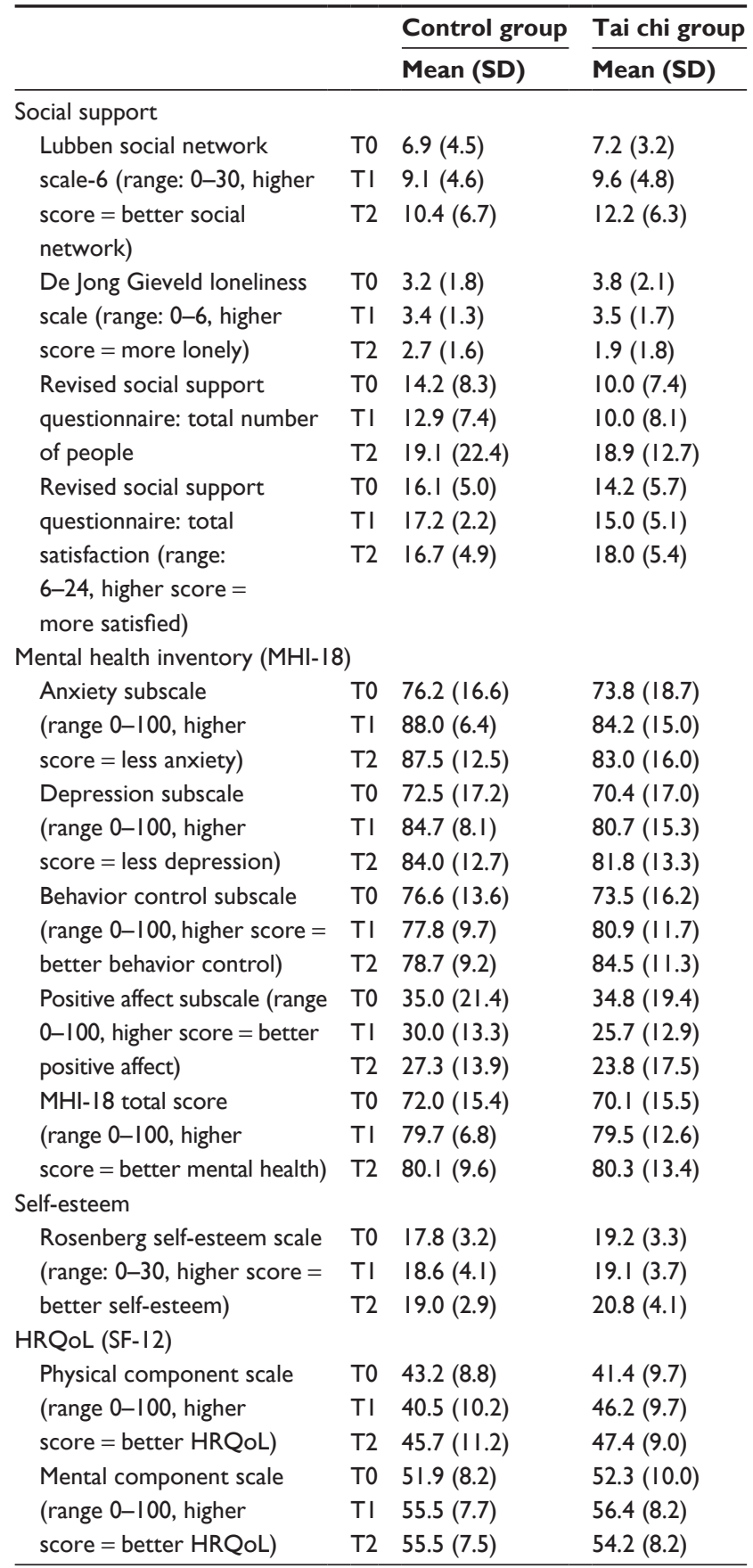

Notes: T0, beginning of the study; TI, three months post-intervention; and T2, six months post-intervention.

Abbreviations: SD, standard deviation; SF-12, I2-Item Short Form Health Survey; HRQoL, health-related quality of life.

During the program evaluation, the participants said they had enjoyed the tai chi qigong classes, and $82 \%$ would continue the exercises. No injury or discomfort was reported from the tai chi qigong sessions. Nine people said their social network had broadened after joining the classes and that they had made more friends. 


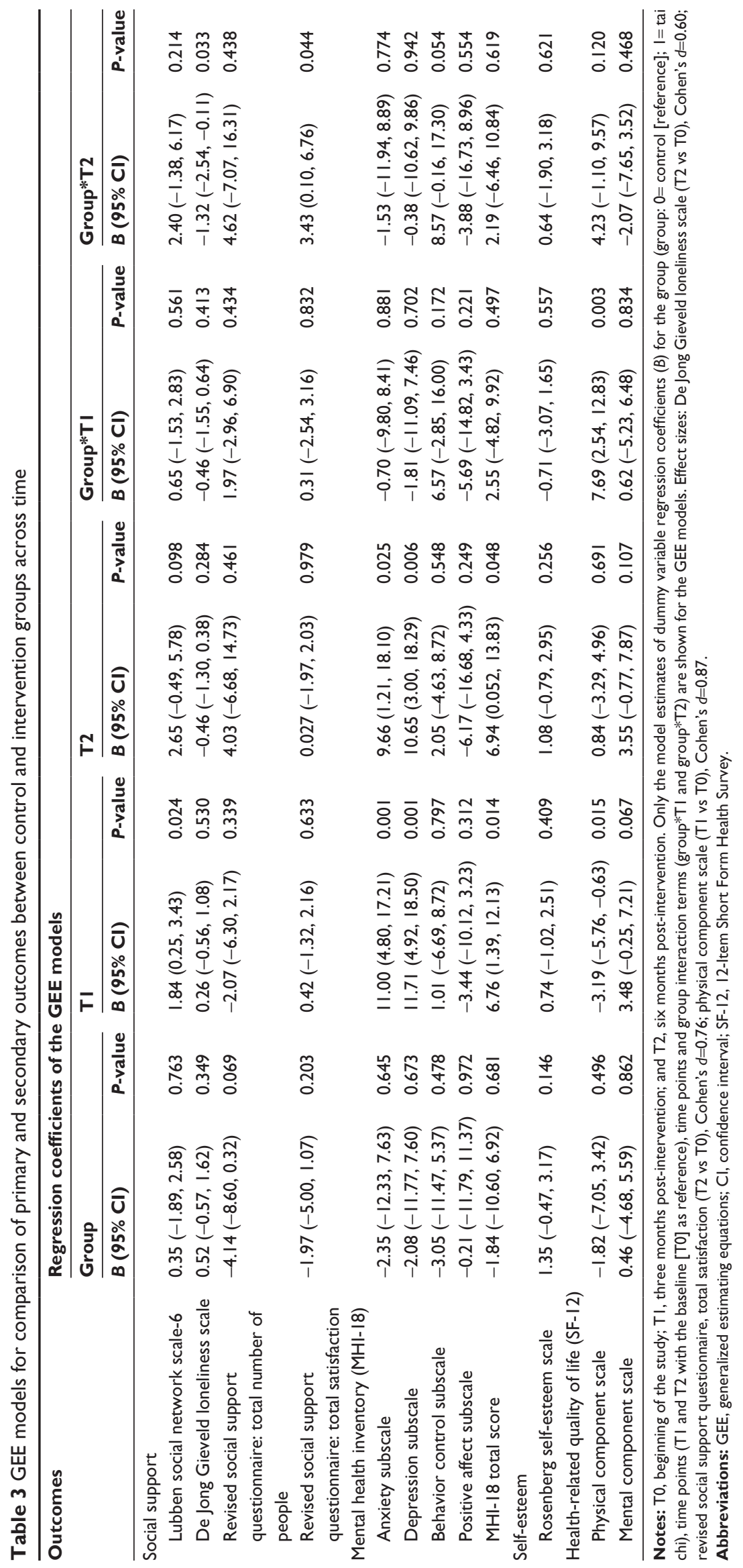




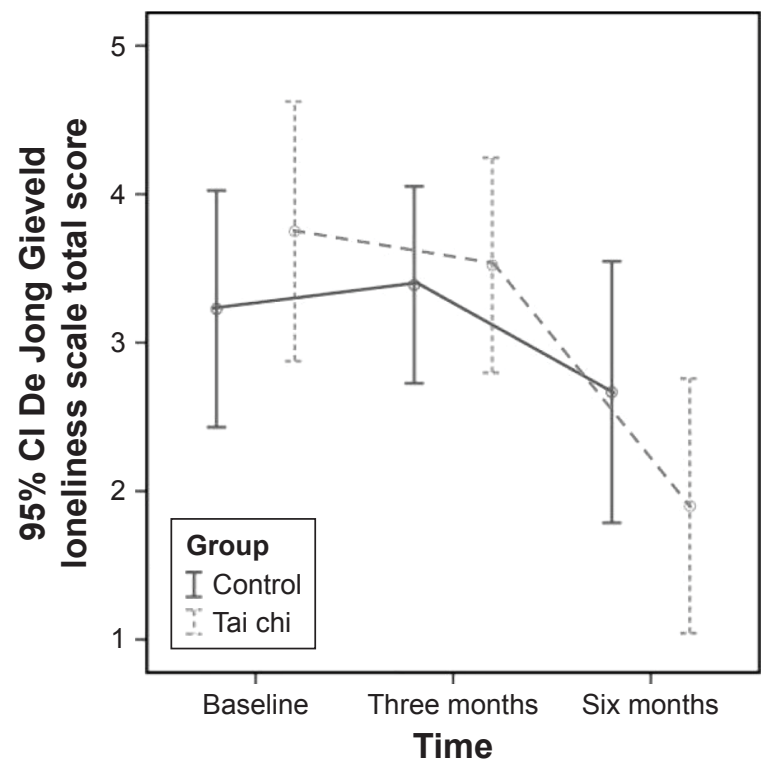

Figure 2 De Jong Gieveld loneliness scale across time between control and tai chi qigong groups.

Abbreviation: $\mathrm{Cl}$, confidence interval.

A total of eight health ambassadors (three male and five female) were recruited. Their age ranged from 65 to 81 years with an average of 73.5 years. Their feedback on participating in the study was very positive, and they had enjoyed practicing tai chi qigong with the hidden elderly. They said that the study was not only beneficial to the hidden elderly in other ways, but also enhanced their health through doing the exercises. They mentioned that the major barriers for the elderly in practicing tai chi qigong were their poor memory

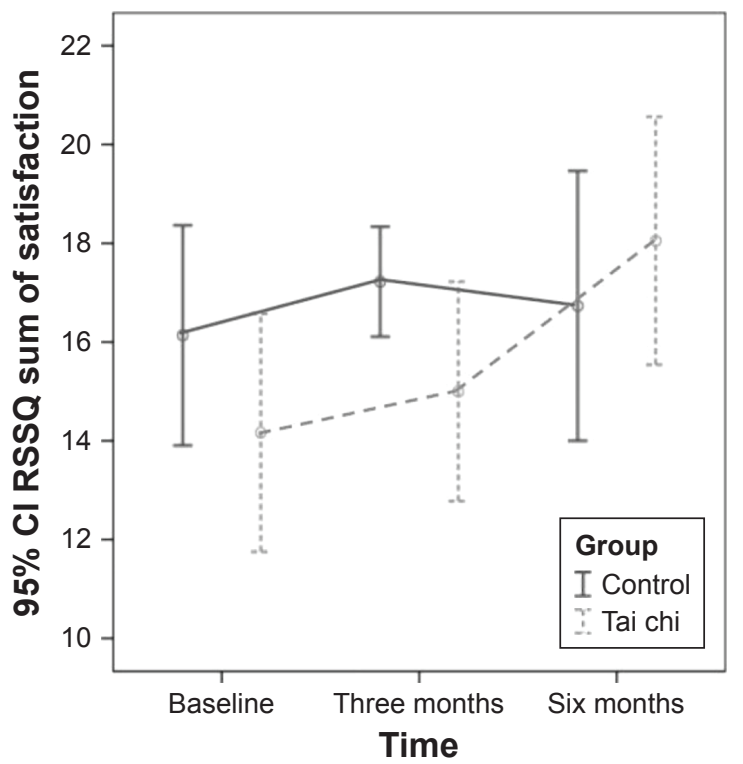

Figure 3 RSSQ sum of satisfaction across time between control and tai chi qigong groups.

Abbreviations: RSSQ, revised social support questionnaire; $\mathrm{Cl}$ confidence interval.

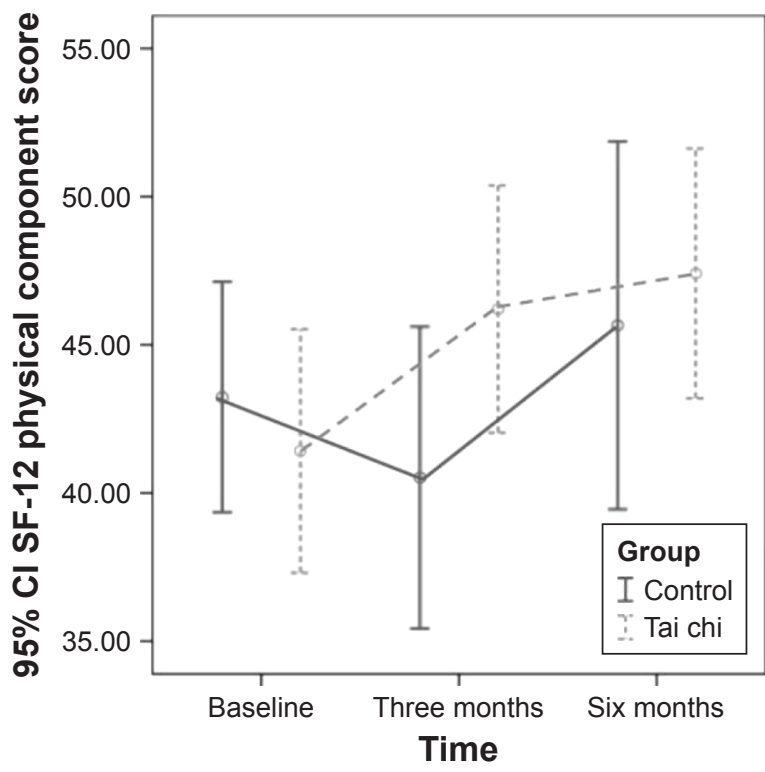

Figure 4 Physical component scales across time between control and tai chi qigong groups.

Abbreviations: SF-12, I2-Item Short Form Health Survey; Cl confidence interval.

and muscle weakness, and suggested that daily practice could help to overcome such barriers.

\section{Discussion}

The hidden elderly form a group of older adults disengaged from the community and with a poor network of family or friends. The study implemented a tai chi qigong program using an elderly neighborhood volunteer approach that aimed to provide social contacts for the hidden elderly and motivate their participation. Results revealed positive effects in social support outcomes and in the physical health of the tai chi qigong group. The social support outcomes included a broadened social network, reduced feelings of loneliness, and increased satisfaction with perceived social support. The improvement in physical health also reflects what the literature identifies as the positive effects of tai chi qigong on loneliness and perceived social support from friends. ${ }^{13,38}$ No adverse effects were reported from the tai chi qigong classes indicating it was a safe form of exercise for the elderly.

During the first three months, participants in the two study groups did not show significant changes in the loneliness scale and satisfaction of social support. However, decreased feeling of loneliness and increased satisfaction in social support were noted in the tai chi qigong group at the sixth month's follow-up assessment. This might be because the tai chi qigong participants continued their daily self-practice with peer volunteers in groups after the completion of tai chi qigong program, and they eventually became friends, 
and thus, they felt less lonely and perceived supports from peer volunteers after six months. This result matched with a previous study which showed that time was needed build up rapport with friends. ${ }^{13}$ On the other hand, these three outcome variables in the control group showed reverse trends between the first and second three-month period. Indeed, these were results of a relatively small-scale study; a large-scale study would be needed to confirm their trends.

Age has been found to be negatively related to network size, ${ }^{3}$ but most public policies are concerned only with the physical issues of aging. However, social issues, such as isolation, are important because they are linked to poor physical and mental health. ${ }^{39}$ The concept of social isolation refers to a lack of social integration. ${ }^{40}$ Both morbidity and mortality are higher among more socially isolated and lonelier older people. ${ }^{41}$ The consequences of such include poor health, depression, personal disorders, and suicide. ${ }^{42}$ Given the association between aging and loss of social contacts, these negative consequences make the prevention and treatment of social isolation an important priority in aging populations. ${ }^{43}$ In the present pilot study, practicing tai chi qigong in a group provided social contacts for the hidden elderly, and its positive effects were reflected in the significantly reduced feelings of loneliness and increased satisfaction with social support the group showed. A well-received physical activity intervention of this kind also added physical health benefits to those of the social intervention.

Social integration is essential to successful aging. ${ }^{3}$ Previous studies have indicated that older people find social connectedness and activity are strongly associated with overall health. ${ }^{44}$ Older people who maintain outgoing leisure behavior have higher rates of well-being, and remaining socially active is likely to alleviate loneliness. ${ }^{43}$ The tai chi qigong program in this study aimed specifically to increase social integration for older people and to facilitate their connection with other people in the community, a strategy that can help to reduce the loss of physical and mental health associated with social isolation. ${ }^{42}$ This pilot study provided evidence that tai chi qigong was an effective intervention, offering social activity in a group format. Dickens et al also claimed that activities offered at the group level are more likely to be beneficial than one-to-one interventions. ${ }^{11}$ Participatory interventions combining social activity and support are likely to be beneficial as well. ${ }^{11}$

When planning an intervention for hidden elderly, it was found that the major challenge was a lack of motivation to participate in the program. The literature suggests that social support for older adults, having active friends or being encouraged by at least one person, is the most influential stimulus to take part in activities. ${ }^{45}$ Stimulating participation in social activities may therefore be an effective tool to reduce social isolation and loneliness among older adults. Utilizing elderly neighborhood volunteers to mobilize hidden elderly people and strengthen their social networks was an effective solution. The health ambassadors appeared to have motivated the hidden elderly to participate in this study. The dropout rate was much lower in the tai chi qigong group than in the control $(4.2 \%$ vs $18.2 \%$ at three months and $16.7 \%$ vs $31.8 \%$ at six months), and this could have been due to the ambassadors, who were considered as facilitators in motivating the hidden elderly to take part in the program. According to Cheung and Ngan, the contribution of volunteer networking greatly helps the social integration of isolated and frail elderly, whose social networks are empowered in this way. ${ }^{46}$ The role of volunteers as a source of informal support is remarkable, and the assistance of well-trained elderly neighborhood volunteers is likely to have enhanced the motivation of hidden elderly people to participate in the present study.

Volunteering provides a socially rewarding and physically active environment that greatly benefits seniors. ${ }^{47}$ Most elderly people who volunteer invest more hours than younger people because they have more uncommitted time,${ }^{48}$ and their participation is a vehicle by which residents can increase individual and collective capacities as well as connections with their neighbourhood. ${ }^{49}$ This is an effective strategy that engages older adults in neighborhood improvement, and volunteers provide immediacy and personal affirmation of informal social support. Volunteers of this kind are widely available and easily accessible in the community, having a strong psychological sense of neighborliness and commitment to their communities, and also expressing a high level of integration into the community.

In summary, social isolation is modifiable through social connections, which reduce loneliness. Innovative interventions to deal with social isolation and loneliness should be conducted and evaluated because of the magnitude of the health risk among the hidden elderly.$^{50}$ By understanding causal mechanisms, policymakers and researchers will be able to develop specific and appropriate strategies for such people.

\section{Limitations}

This pilot study has several limitations. First, the tai chi qigong program used neighborhood volunteer support, and 
it is unclear whether the positive results were due to this support or to the tai chi qigong practice itself. Future studies may consider adding a tai chi qigong only group, without the volunteer support. Second, the recruitment process relied heavily on social workers to identify the hidden elderly, so only those recognized by the social workers were recruited. Selection bias can be reduced by recruiting hidden elderly openly from the community, with the support of neighborhood volunteers and the community outreach team. Third, the small sample size in this pilot study may decrease its statistical power and reduce the chances of detecting true effects. Therefore, these findings must be interpreted with caution as they may not be generalizable to a larger population. Finally, all the outcome variables were obtained via questionnaires, which are subjective measures. Future studies may consider adding objective measures, such as social network size or physical fitness, to increase the variety of outcome measures.

\section{Conclusion}

With the life expectancy of older people rising, the aging population is an issue of some alarm, and has important implications for the provision of health services. Social isolation is the most typical situation of the hidden elderly, but when tai chi qigong exercises are performed in a group, the reinforcement that participation brings can have social benefits to counter isolation and create friendships. This pilot study confirms that tai chi qigong, with the help of elderly neighborhood volunteers, can be a feasible social intervention for hidden elderly. Its potential benefits in improving such people's social and psychological health suggest there is a need for a full-scale RCT to reveal the full empirical effects.

\section{Acknowledgments}

This study was funded by the School Seed Grant from the Nethersole School of Nursing, the Chinese University of Hong Kong. The authors thank the social workers of the Christian Family Service Centre for assisting in the study. Partial preliminary results at three months were presented in the Lancet-CMS conference in October $2016 .^{51}$

\section{Author contributions}

All authors contributed toward data analysis, drafting and revising the paper, and agree to be accountable for all aspects of the work.

\section{Disclosure}

The authors report no conflicts of interest in this work.

\section{References}

1. World Health Organization. 10 facts on ageing and health. 2015 [updated September 2015]. Available from: http://www.who.int/ features/factfiles/ageing/en/. Accessed September 2, 2016.

2. Steering Committee on Population Policy. Population policy: strategies and initiatives. Hong Kong SAR: Chief Secretary for Administration's Office; 2015.

3. Cornwell B, Laumann EO, Schumm LP. The social connectedness of older adults: a national profile. Am Sociol Rev. 2008;73(2):185-203.

4. Hong Kong Special Administrative Region Government News. Press releases. Assistance to the unidentified elderly [updated 2007]. Available from: http://www.info.gov.hk/gia/general/200703/28/P200703280174. htm. Accessed August 31, 2016.

5. Asia-Pacific Institute of Ageing Studies, Lingnan University. Hidden elderly service research [updated 2009]. Available from: http://webphoto. ln.edu.hk/thumbnails.php?album=399. Accessed August 31, 2016.

6. The Chinese University of Hong Kong. A Qualitative Study on 'Hidden Elderly' in Hong Kong. Hong Kong Special Administrative Region: Central Policy Unit; 2008.

7. Chau PH, Gusmano MK, Cheng JO, Cheung SH, Woo J. Social vulnerability index for the older People-Hong Kong and New York city as examples. J Urban Health. 2014;91(6):1048-1064.

8. Eng PM, Rimm EB, Fitzmaurice G, Kawachi I. Social ties and change in social ties in relation to subsequent total and cause-specific mortality and coronary heart disease incidence in men. Am J Epidemiol. 2002;155(8): 700-709.

9. Cattan M, White M, Bond J, Learmouth A. Preventing social isolation and loneliness among older people: a systematic review of health promotion interventions. Ageing Soc. 2005;25(1):41-67.

10. Greaves CJ, Farbus L. Effects of creative and social activity on the health and well-being of socially isolated older people: outcomes from a multi-method observational study. J R Soc Promot Health. 2006;126(3): 134-142.

11. Dickens AP, Richards SH, Greaves CJ, Campbell JL. Interventions targeting social isolation in older people: a systematic review. $B M C$ Public Health. 2011;11:647.

12. Saito T, Kai I, Takizawa A. Effects of a program to prevent social isolation on loneliness, depression, and subjective well-being of older adults: a randomized trial among older migrants in Japan. Arch Gerontol Geriatr. 2012;55(3):539-547.

13. Chan AW, Lee A, Lee DT, Sit JW, Chair SY. Evaluation of the sustaining effects of Tai Chi Qigong in the sixth month in promoting psychosocial health in COPD patients: a single-blind, randomized controlled trial. Scientific World J. 2013;2013:425082.

14. Lee LY, Lee DT, Woo J. The psychosocial effect of Tai Chi on nursing home residents. J Clin Nurs. 2010;19(7-8):927-938.

15. Lu X, Hui-Chan CW, Tsang WW. Tai Chi, arterial compliance, and muscle strength in older adults. Eur J Prev Cardiol. 2013;20(4):613-619.

16. Ni GX, Song L, Yu B, Huang CH, Lin JH. Tai Chi improves physical function in older Chinese women with knee osteoarthritis. J Clin Rheumatol. 2010;16(2):64-67.

17. Wayne PM, Walsh JN, Taylor-Piliae RE, et al. Effect of tai chi on cognitive performance in older adults: systematic review and meta-analysis. J Am Geriatr Soc. 2014;62(1):25-39.

18. DiGiacomo M, Lam P, Roberts BL, Lau TC, Song R, Davidson PM. Exploring the reasons for adherence to t'ai chi practice. $J$ Altern Complement Med. 2010;16(12):1245-1246.

19. Chan AW, Lee A, Lee DT, et al. The sustaining effects of Tai chi Qigong on physiological health for COPD patients: a randomized controlled trial. Complement Ther Med. 2013;21(6):585-594.

20. Ng SM, Wang CW, Ho RT, et al. Tai chi exercise for patients with heart disease: a systematic review of controlled clinical trials. Altern Ther Health Med. 2012;18(3):16-22.

21. Sun J, Buys N, Jayasinghe R. Effects of community-based meditative Tai Chi programme on improving quality of life, physical and mental health in chronic heart-failure participants. Aging Ment Health. 2014;18(3): 289-295. 
22. Zhang Y, Fu FH. Effects of 14-week Tai Ji Quan exercise on metabolic control in women with type 2 diabetes. Am J Chin Med. 2008;36(4): 647-654.

23. Wolf SL, O'Grady M, Easley KA, Guo Y, Kressig RW, Kutner M. The influence of intense Tai Chi training on physical performance and hemodynamic outcomes in transitionally frail, older adults. J Gerontol A Biol Sci Med Sci. 2006;61(2):184-189.

24. Field T. Tai chi research review. Complement Ther Clin Pract. 2011; 17(3):141-146.

25. Chan AW, Yu DS, Choi KC, Lee DT, Sit JW, Chan HY. Tai chi qigong as a means to improve night-time sleep quality among older adults with cognitive impairment: a pilot randomized controlled trial. Clin Interv Aging. 2016;11:1277-1286.

26. Haski-Leventhal D, Bargal D. The volunteer stages and transitions model: organizational socialization of volunteers. Hum Relat. 2008; 61(1):67-102.

27. Haski-Leventhal D, Ben-Arieh A, Melton GB. Between neighborliness and volunteerism: participants in the strong communities initiative. Fam Community Health. 2008;31(2):150-161.

28. Browne RH. On the use of a pilot sample for sample size determination. Stat Med. 1995;14(7):1933-1940.

29. Lubben JE. Assessing social networks among elderly populations. Fam Community Health. 1988;11(3):42-52.

30. Rubinstein RL, Lubben JE, Mintzer JE. Social isolation and social support: an applied perspective. J Appl Gerontol. 1994;13(1):58-72.

31. Yunong H. Family relations and life satisfaction of older people: a comparative study between two different hukous in China. Ageing Soc. 2012;32(1):19-40

32. Leung GT, de Jong Gierveld J, Lam LC. Validation of the Chinese translation of the 6-item De Jong Gierveld Loneliness Scale in elderly Chinese. Int Psychogeriatr. 2008;20(6):1262-1272.

33. Sarason IG, Sarason BR, Shearin EN, Pierce GR. A brief measure of social support: practical and theoretical implications. J Soc Pers Relat. 1987;4(4):497-510.

34. Veit CT, Ware JE Jr. The structure of psychological distress and well-being in general populations. J Consult Clin Psychol. 1983;51(5):730-742.

35. Schmitt DP, Allik J. Simultaneous administration of the Rosenberg Self-Esteem Scale in 53 nations: exploring the universal and culturespecific features of global self-esteem. J Pers Soc Psychol. 2005;89(4): 623-642.

36. Lam ET, Lam CL, Fong DY, Huang WW. Is the SF-12 version 2 health survey a valid and equivalent substitute for the SF-36 version 2 health survey for the Chinese? J Eval Clin Pract. 2013;19(1):200-208.
37. Hardin JW. Generalized estimating equations (GEE). In: Everitt BS, Howell DC, editors. Encyclopedia of Statistics in Behavioral Science. Volume 2. Wiley Online Library; 2005:721-729.

38. Park YJ, Park IH. Effect of tai chi exercise on loneliness, sleep pattern, and instrumental activities of daily living in elderly women. J Muscle Jt Health. 2010;17(2):151-161.

39. Chen S, Conwell Y, Chiu HF. Loneliness and aging in China - a public health problem in need of solutions. Int Psychogeriatr. 2014;26(11): 1771-1772.

40. Grenade L, Boldy D. Social isolation and loneliness among older people: issues and future challenges in community and residential settings. Aust Health Rev. 2008;32(3):468-478.

41. Steptoe A, Shankar A, Demakakos P, Wardle J. Social isolation, loneliness, and all-cause mortality in older men and women. Proc Natl Acad Sci US A. 2013;110(15):5797-5801.

42. Pettigrew S. Reducing the experience of loneliness among older consumers. J Res Consum. 2007;12:1-4.

43. Toepoel V. Ageing, leisure, and social connectedness: how could leisure help reduce social isolation of older people? Soc Indic Res. 2013; 113(1):355-372.

44. Giummarra MJ, Haralambous B, Moore K, Nankervis J. The concept of health in older age: views of older people and health professionals. Aust Health Rev. 2007;31(4):642-650.

45. Orsega-Smith EM, Payne LL, Mowen AJ, Ho C, Godbey GC. The role of social support and self-efficacy in shaping the leisure time physical activity of older adults. J Leis Res. 2007;39(4):705.

46. Cheung CK, Ngan MH. Contributions of volunteer networking to isolated seniors in Hong Kong. J Gerontol Soc Work. 2000;33(2):79-100.

47. McDonald TW, Chown EL, Tabb JE, Schaeffer AK, Howard EK. The impact of volunteering on seniors' health and quality of life: an assessment of the retired and senior volunteer program. Psychology. 2013; 4(3):283.

48. Anukul C. Depression and Social Cohesion: Is There a Connection? [master's thesis]. Long Beach, CA: California State University; 2014.

49. Ohmer ML. Citizen participation in neighborhood organizations and its relationship to volunteers' self- and collective efficacy and sense of community. Soc Work Res. 2007;31(2):109-120.

50. Coyle CE, Dugan E. Social isolation, loneliness and health among older adults. J Aging Health. 2012;24(8):1346-1363.

51. Chan AWK, Yu DSF, Choi KC, Chan HYL, Wong EML. Effects of a peer-assisted tai chi qigong program on social isolation and psychological well-being in Chinese hidden elders: a pilot randomised controlled trial. The Lancet. 2016;388(Suppl 1):S23. 


\section{Supplementary material}

Table SI The 18 movements of tai chi qigong
I. Commencing form and regulating breathing
2. Expanding your chest
3. Painting a rainbow
4. Circling arms separate the clouds
5. Fixed step invert brachial
6. Row a boat in the middle of the lake
7. Shoulder the ball
8. Turn the body to look at the moon
9. Twisting waist and pushing palms
10. Horse step and cloud hands
II. Drag the sea and watch the sky
12. Undulating waves
13. Dove spreading wings
14. Extend arms and punch
15. Wild goose flying
16. Flywheel turning
17. Bounce ball while stepping
18. Pressing palms in calmness

\section{Publish your work in this journal}

Clinical Interventions in Aging is an international, peer-reviewed journal focusing on evidence-based reports on the value or lack thereof of treatments intended to prevent or delay the onset of maladaptive correlates of aging in human beings. This journal is indexed on PubMed Central, MedLine,
CAS, Scopus and the Elsevier Bibliographic databases. The manuscript management system is completely online and includes a very quick and fair peer-review system, which is all easy to use. Visit http://www.dovepress. com/testimonials.php to read real quotes from published authors. 